\title{
Index to Volume IV
}

The alphabet and orthography of Byelorussian in the 20th century. By P. J. Mayo, 1:28

Die älteste ostslawische Kunstdichtung 1575-1647. Ed. by $H$. Rothe, review (A. B. McMillin) 1:58

Barysienka, V. et al. (eds) Janka Kupała. Zbor tvoraŭ, vols $1-7$, review (A. Nadson) 2:74

Bas, I. Litaraturaznaŭčyja eciudy, review (A. B. McMillin) 2:70

Bergman, A. Rzecz o Bronislawie Taraszkiewiczu, review (J. Dingley) $2: 71$

BIEDER, H. Die morphologische Adaptation deutscher Substantiva in altweißrussischen Sprachdenkmälern aus der Sicht der deutschen historischen Morphologie, $2: 3$

Biełaruskaja Savieckaja Encykłapiedyja. Ed. by P. Broŭka et al., review (A. Nadson) 3/4:157

The Byelorussian impact on Karaite and Yiddish. By P. Wexler, 3/4:99

Byelorussian orthography: from the 1933 reform to the present day. By P. J. Mayo, 2:25

Canturija, V. A. Istorija architektury Belorussii, review (L. A. J. Hughes) 2:72

Castotny sloŭnik bielaruskaj movy. Mastackaja proza. By N. S. Mažejka, A. Ja. Suprun, review (P. J. Mayo) 1:51

Demografičeskije problemy razvitija Minska. By S. A. Pol'skij, review (J. Sallnow) 1:56

DINGLEY, J. Some recent Soviet publications on Francisk Skaryna (review article), 3/4:148

[reviews:]

Bergman, A. Rzecz o Bronistawie Taraszkiewiczu, 2:71

Maleckij, J. Pad znakam Pahoni. Uspaminy, 1:55

Rjabcevič, V. N. O čem rasskazyvajut monety, 3/4:164

Fair Land of Byelorussia: An Anthology of Modern Byelorussian Poetry. Trans. by W. May, with Intro. by Maksim Tank, review (A. B. McMillin) $1: 50$

du Feu, V. M. [review:]

Padłužny, A. I. Narys akustyčnaj fanietyki bielaruskaj movy, 2:75
German lexical borrowings in the Chronicle of Barkulabava and the Memoirs of Theodore Jeŭłašeŭski, By R. J. Patry-Tamushanski, 3/4:112

Gifford, $\mathrm{H}$. [review:]

McMillin, A. B. A History of Byelorussian Literature. From its Origins to the Present Day, 1:53

A History of Byelorussian Literature. From its Origins to the Present Day. By A. B. McMillin, review (H. Gifford) 1:53

Hłybinny, U. Paeta z Božaj laski. Uspaminy $i$ rozdum pra Jazepa Pušču (1902-1964), review (A. B. McIillin) $3 / 4: 161$

Hughes, L. A. J. [review:] Canturija, V. A. Istorija architektury Belorussii, 2:72

The influence of non-linguistic factors on the rise and fall of the Old Byelorussian literary language. By A. G. Waring, 3/4:129

Istorija architektury Belorussii. By V. A. Canturija, review (L. A. J. Hughes) 2:72

Kaleśnik, U. Vietrazi Adysieja. Uładzimir Žyłka $i$ ramantyčnaja tradycyja $\breve{u}$ bielaruskaj paezii, review (A. B. McMillin) 3/4:162

Kłyška, M. K. Stoŭnik sinonimaŭ $i$ blizkaznačnych słoŭ, review (P. J. Mayo) 1:51

Kupała, Janka. Zbor tvoraŭ, vols 1-7. Ed. by V. Barysienka et al., review (A. Nadson) 2:74

Litaraturaznaǔčyja eciudy. By I. Bas, review (A. B. McMillin) 2:70

Majsiejenka, A. F. Tvorčaść Michasia Zareckaha: Stanauleńnie talentu, review (A. B. McMillin) 3/4:163

Malecki, J. Pad znakam Pahoni. Uspaminy, review (J. Dingley) 1:55

May, W. (trans.). Fair Land of Byelorussia: An Anthology of Modern Byelorussian Poetry, review (A. B McMillin) 1:50

MAYO, P. J. The alphabet and orthography of Byelorussian in the 20th century, 1:28

Byelorussian orthography: from the 1933 reform to the presen: day, $2: 25$

[reviews:]

Kłyška, M. K. Stoŭnik sinonimaŭ 
i blizkaznačnych stoŭ, 1:51

Mažejka, N. S., Suprun, A. Ja. Castotny stoŭnik bietaruskaj movy. Mastackaja proza, 1:51

Mažejka, N. S., Suprun, A. Ja. Častotny stoǔnik bielaruskaj movy. Mastackaja proza, review (P. J. Mayo) 1:51

McMILLIN, A, B. [with Vera Rich]. Taras na Parnasie, translated and annotated, 1:9

[reviews:]

Bas, I. Litaraturaznaŭčyja eciudy, $2: 70$

Fair Land of Byelorussia: An Anthology of Modern Byelorussian Poetry, 1:50

Hłybinny, U. Paeta z Božaj laski. Uspaminy $i$ rozdum pra Jazepa Pušču (1902-1964). 3/4:161

Kaleśnik, U. Vietrazi Adysieja. Utadzimir Žyłka $i$ ramantyčnaja tradycyja $\breve{u}$ bielaruskaj paezii, $3 / 4: 162$

Majsiejenka, A. F. Tvorčaść Michasia Zareckaha: Stanaǔleńnie talentu, 3/4:163

Rothe, H. (ed.). Die älteste ostslawische Kunstdichtung 1575$1647, \quad 1: 58$

Smykoŭskaja, V. I. Tvorčaja kancepcyja piśmieńnika: Zaduma $i$ jaje mastackaje ŭvasableńnie $\breve{u}$ "Paleskaj chronicy" I. Mieleža, $1: 60$

McMillin, A. B. A History of Byelorussian Literature. From its $\mathrm{Or}$ igins to the Present Day, review (H. Gifford) 1:53

Die morphologische Adaptation deutscher Substantiva in altweißrussischen Sprachdenkmälern aus der Sicht der deutschen historischen Morphologie. By $\mathrm{H}$. Bieder, 2:3

IJADSON, A. Selected bibliography on Byelorussia, 1976, 1:61

Selected bibliography on Byelorussia, 1977, 2:77

Selected bibliography on Byelorussia, 1978-1979, 3/4:166

[reviews:]

Biełaruskaja Savieckaja Encykłapiedyja, ed. P. Broŭka et al., 3/4:157

Kupała, Janka. Zbor tvoraŭ, vols 1-7, ed. by V. Barysienka et al., $2: 74$

Sipovič, ¿. (ed.). The Pontifical Liturgy of Saint John Chrysostom, $3 / 4: 165$

Narys akustyčnaj fanietyki biełaruskaj movy. By A. I. Padłužny, review (V. M. du Feu) 2:75
The Niaśviž estates of Prince Nicholas Christopher Radziwiłk: the formation of a Byelorussian latifundium (1565-1616). By M. Siekierski, 2:48

$O$ čem rasshazyvajut monety. By V. N. Rjabcevič, review (J. Dingley) $3 / 4: 164$

Pad znakam Pahoni. Uspaminy. By J. Malecki, review (J. Dingley) 1:55

Padłužny, A. I. Narys akustyčnaj fanietyki bielaruskaj movy, review (V. M. du Feu) 2:75

Paeta z Božaj laski. Uspaminy $i$ rozdum pra Jazepa Pušč (1902-1964). By U. Hłybinny, review (A. B. McMillin) 3/4:161

PATRY-TAMUSHANSKI, R. J. German lexical borrowings in the Chronicle of Barkutabava and the Memoirs of Theodore Jeŭłašeǔski, $3 / 4: 112$

Pol'skij, S. A. Demografičeskije problemy razvitija Minska, review (J. Sallnow) 1:56

The Pontifical Liturgy of Saint John Chrysostom. Ed. and annot. by C. Sipovič, review (A. Nadson) 3/4:165

RICH, Vera [with A. B. McMillin]. Taras na Parnasie, translated and annotated, $1: 9$

Rjabcevič, V. N. O čem rasskazyvajut monety, review (J. Dingley) 3/4: 164

Rothe, H. (ed.). Die älteste ostslawische Kunstdichtung 1575-1647, review (A. B. McMillin) 1:58

Rothe, H. (ed.). Die älteste ostslawische Kunstdichtung 1575-1647, review (A. B. McMillin) 1:58

Rzecz o Bronisławie Taraszkiewiczu. By A. Bergman, review (J. Dingley) $2: 71$

Sallnow, J. [review:]

Pol'skij, S. A. Demografičeskije problemy razvitija Minska, 1:56

Selected bibliography on Byelorussia, 1976, 1:61

Selected bibliography on Byelorussia, 1977, 2:77

Selected bibliography on Byelorussia, 1978-1979, 3/4:166

SIFKIERSKI, M. The Niaśviž estates of Prince Nicholas Christopher Radziwiłł: the formation of a Byelorussian latifundium (15651616), 2:48

Theodore Jeŭłašeŭski and his Memoirs, 1:5

Sipovič, C. (ed.). The Pontifical Liturgy of Saint John Chrysostom, review (A. Nadson) 3/4:165 
Słoŭnik sinonimaŭ $i$ blizkaznačnych stoŭ. By M. K. Kłyška, review (P. J. Mayo) 1:51

Smykoŭskaja, V. I. Tvorčaja kancepcyja piśmieńnika: Zaduma $i$ jaje mastackaje ŭvasableńnie $\breve{u}$ "Paleskaj chronicy" I. Mieleža, review (A. B. McMillin) 1:60

Some recent Soviet publications on Francisk Skaryna (review article). By J. Dingley, 3/4:148

Taras na Parnasie. Translated and annotated by A. B. McMillin and Vera Rich, 1:9

Theodore Jeŭłašeŭski and his Memoirs. By M. Siekierski, 1:5

Tvorčaja kancepcyja piśmieńnika: Zaduma $i$ jaje mastackaje ŭvasab- leńnie $\breve{u}$ "Paleskaj chronicy" I. Mieleža. By V. I. Smykoŭskaja, review (A. B. McMillin) 1:60

Tvorčaść Michasia Zareckaha: Stanaŭlennie talentu. By A. F. Majsiejenka, review (A. B. McMillin) $3 / 4: 163$

Vietrazi Adysieja. Utadzimir Žytka i ramantyčnaja tradycyja $\breve{u}$ biełaruskaj paezii. By U. Kaleśnik, review (A. B. McMillin) 3/4:162

WARING, A. G. The influence of nonlinguistic factors on the rise and fall of the Old Byelorussian literary language, 3/4:129

WEXLER, $P$. The Byelorussian impact on Karaite and Yiddish, 3/4: 99 